\title{
AUTOMATED REFLECTANCE TARGET DETECTION FOR AUTOMATED VICARIOUS RADIOMETRIC CORRECTION OF UAV IMAGES
}

\author{
Seunghwan Ban ${ }^{1}$, Taejung Kim ${ }^{1,2}$ \\ 1 Program in Smart City Engineering, Inha University, Incheon, S.Korea - 22211349@inha.edu \\ 2 Dept.of Geoinformatic Engineering, Inha University, Incheon, S.Korea - tezid@inha.ac.kr
}

\author{
Commission I, ICWG I/II
}

KEY WORDS: UAV, Radiometric calibration, Reflectance target, Digital number, Canny edge detection, Automatic detection.

\begin{abstract}
:
Recently, with increasing use of unmanned aerial vehicle (UAV), radiometric calibration of UAV images has become an important pre-processing step for application such as vegetation mapping, crop field monitoring, etc. In order to obtain accurate spectral reflectance, some UAVs measure irradiance at the time of image acquisition. However, most of UAV systems do not have such irradiance sensors. In these cases, vicarious radiometric correction method has to be used. Digital numbers (DNs) of imaged ground reflectance targets are measured and spectral reflectance is acquired from with known reflectance values of the targets. For automated vicarious calibration, a technique for automatically detecting image location of ground reflectance targets has been developed. In this study, we report an improved version of automated reflectance target detection and a new semi-automatic reflectance target detection developed. Test results showed that among the 14 reflectance targets, 13 targets were detected with the automatic target detection method. The undetected target was extracted by the proposed semi-automatic target detect method. Additional test was conducted on the remaining targets to confirm the applicability of our semi-automatic target detection method. As a result, other targets were also detected. The proposed automated and semi-automated target detection method can be used for automated vicarious calibration of UAV images.
\end{abstract}

\section{INTRODUCTION}

UAV images are being used with improved aircraft and sensors in various fields. Users can acquire images at any location and time. Accurate pre-processing of images is essential to improve the quality of UAV data. Radiometric correction is important pre-processing to get accurate spectral information from images for applications such as vegetation mapping, crop field monitoring, etc.

In order to obtain accurate spectral reflectance, some UAVs measure irradiance at the time of image acquisition. Recently, compact-sized multispectral image sensor with an irradiance sensor is mounted in a UAV to obtain spectral information (Mathews, 2015). However, most of UAV systems do not have such irradiance sensors and do not provide radiometric correction coefficients. In these cases, vicarious radiometric correction method has to be used. If radiometric correction coefficients are not provided, vicarious radiometric correction method is used. Spectral reflectance can be obtained using targets which known reflectance values. A study has been conducted to convert from DN to reflectance through linear regression analysis (Shin et al, 2020). It is also called empirical line to derive DN for coefficient of reflectance conversion (Wang and Myint, 2015). To convert reflectance using targets, a user needs to set area where target is located in an image. If more targets are used for reflectance conversion, more time is spent for setting target areas. To remove this manual process, a technique for automatically detecting image location of ground reflectance targets has been developed (Shin et al. 2019).

In this study, we report an improved version of automated reflectance target detection and a new semi-automatic reflectance target detection for automated vicarious radiometric correction.

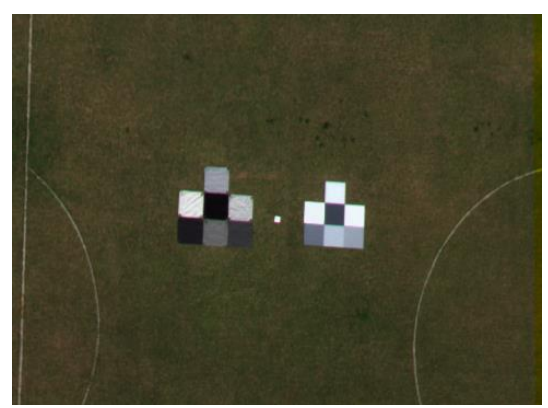

(a)

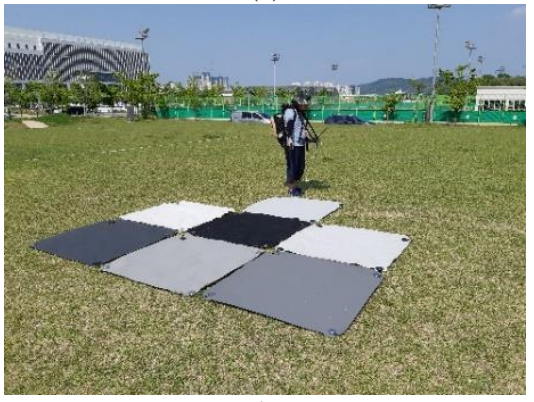

(b)

Figure 1. The reference targets installed for radiometric correction.

\section{DATA AND METHOD}

\subsection{Data Acquisition}

14 Reflectance targets were installed on the grass within a soccer field in Jeonju, South Korea (Figure 1). The reflectance 
targets are specially dyed to have constant reflectance through a spectral range of $435 \mathrm{~nm}$ to $1100 \mathrm{~nm}$. The size of each target was $1.2 \mathrm{~m}$ by $1.2 \mathrm{~m}$. Spectral reflectance of each target was measured using a FieldSpec-3 spectro-radiometer.

UAV images were acquired by a RedEdge-MicaSense camera with an irradiance sensor (Figure 2a). The camera was consisted with 5 bands multispectral camera with 16 bits radiometric resolution, 1280 by 960 pixels and $5.4 \mathrm{~mm}$ focal length. The image was acquired at $100 \mathrm{~m}$ altitude. In this study, we used blue band image among 5 band images for vicarous calibration test (Figure 2b).

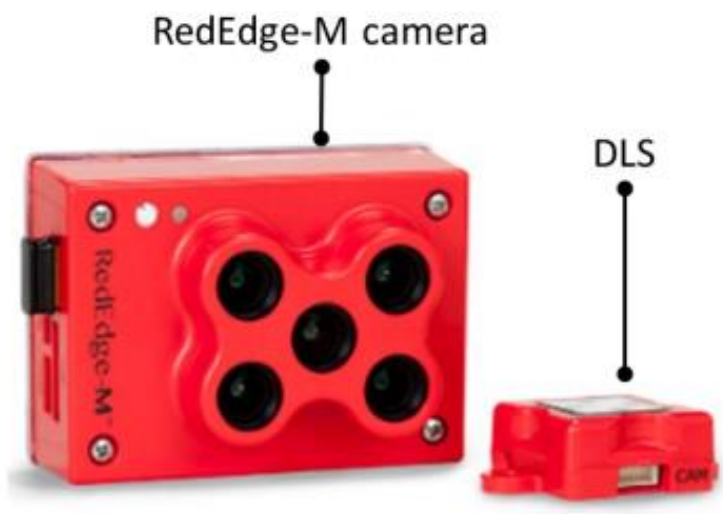

(a)

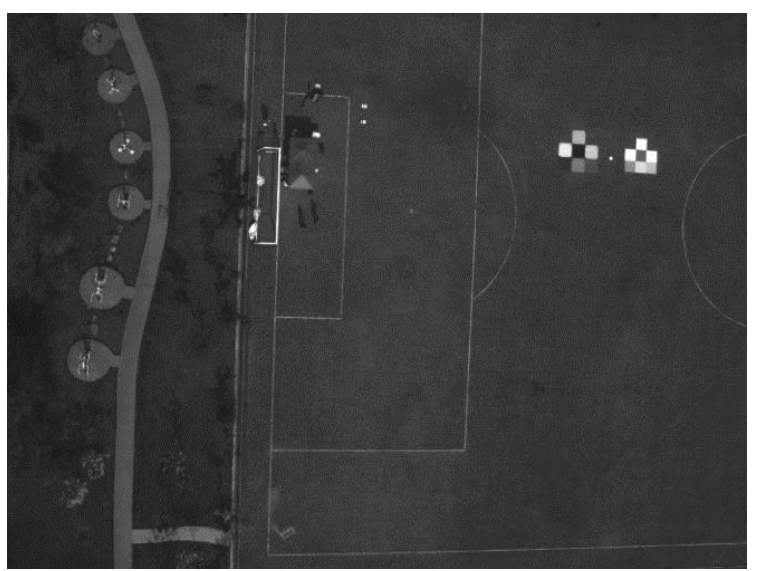

(b)

Figure 2. (a) The multispectral camera and irradiance sensor. (b) Blue band image of Mica-Sense camera.

\subsection{Automatic Target Detect Method}

Our automatic target detection method was composed with four steps (Figure 3). In the first step, homogeneity was calculated through coefficient in a moving window. The moving window had the half size of reference targets. Reference targets are expected to have high homogeneity than natural targets. Therefore, the ratio of standard deviation to mean was used as a measure to extract target candidates. Target candidates were selected if the ratio was less than 0.2. The second step was to extract edge using Canny filter. This was to extract edges of objects with similar and high homogeneity. To enhance the effectiveness of edge extraction, we performed image normalization process. The third step was to reduce the number of target candidates by using the prior knowledge of target size and shape. Thus, we had to know the approximate image GSD and the size of reference target before using automatic target detection method. The final step was the measurement of DN variations among multispectral bands. The reference targets have similar reflectance through visible to near infrared wavelength (Figure 4a). And there is linearity between DN and reflectance (Figure 4b). Therefore, reference targets could be detected from other candidates.

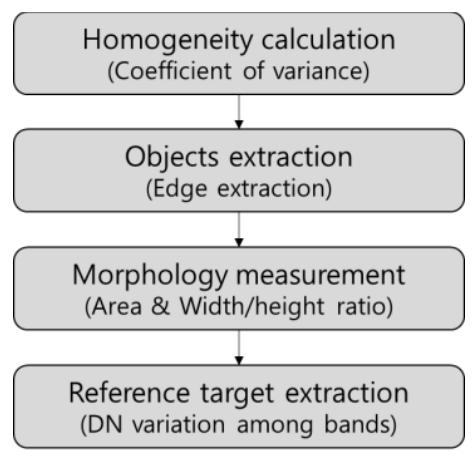

Figure 3. A processing of automatic target detection method.

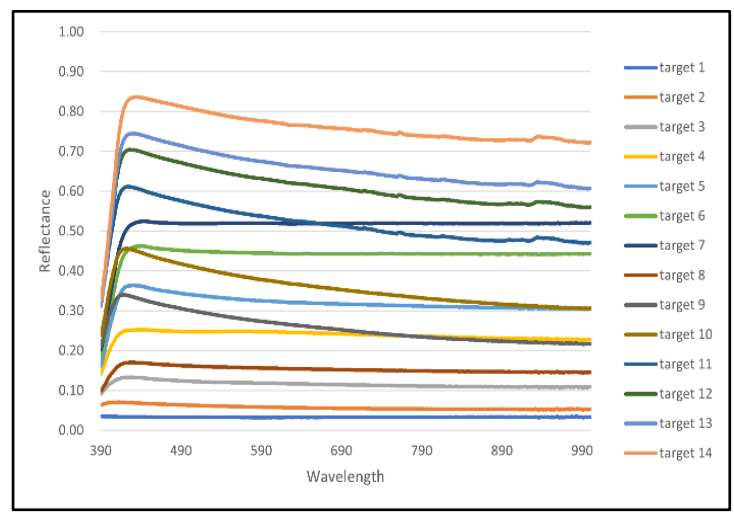

(a)

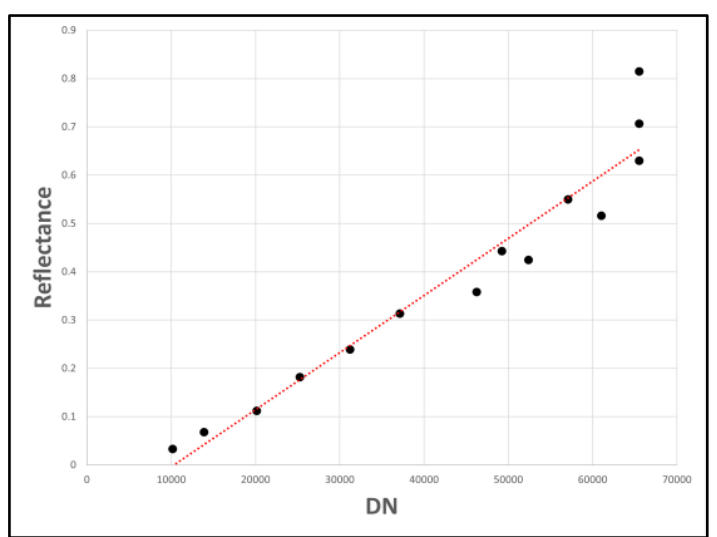

(b)

Figure 4. (a) Spectral reflectance of reference targets (b) Linearity between DN and reflectance of reference targets.

\subsection{Semi-automatic Target Detect Method}

The semi-automatic target detect method repeats automated DN analysis process after receiving initial target location manually (Figure 5). Target area detection is performed by calculating the changes in DN values within the current area of consideration. The area from the initial point is gradually expanded when DN 
values within the area are homogenous. As the area increases, average of DN values of the newly expanded area is calculated (Figure 6a). The area is expanded from manually selected point in four direction (up, down, left and right). The average DN values before and after area expansion are calculated. The expansion stops when the difference average of DN value is greater than the threshold we set (Figure 6b). In this study, the threshold was set to 16 , which corresponds to about $6 \%$ of total $\mathrm{DN}$ value range.

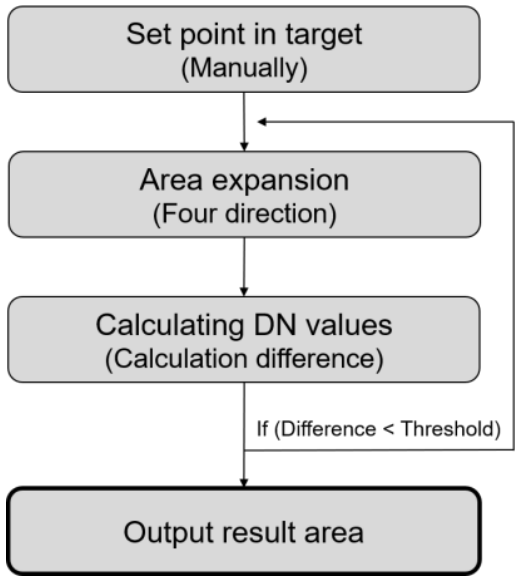

Figure 5. A processing of semi-automatic target detection method.

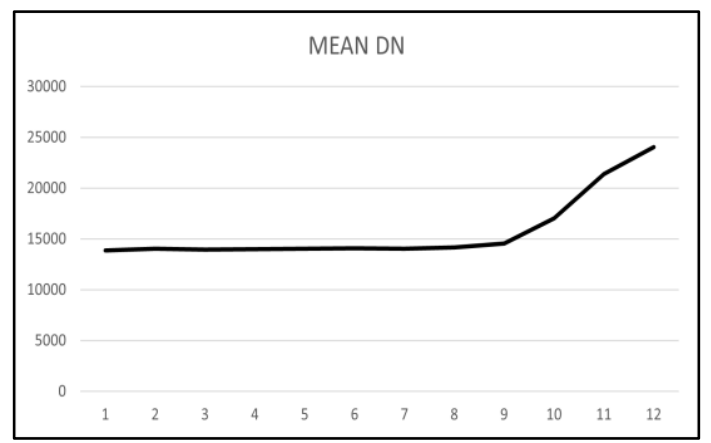

(a)

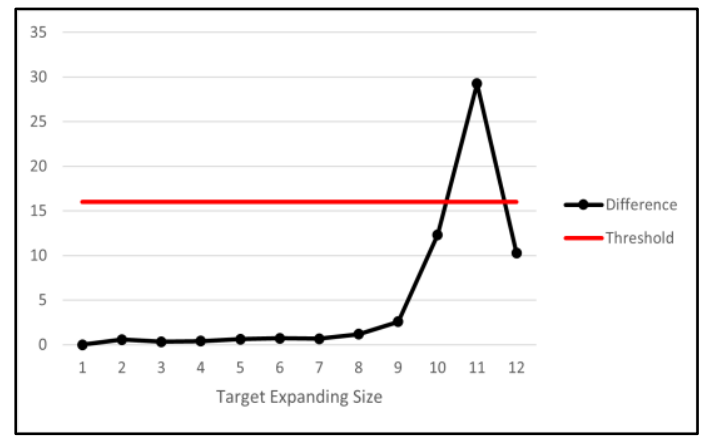

(b)

Figure 6. Example of semi-automatic target detect result. (a) Example of mean DN value with expanding detection size. (b) Example of Difference value with expanding detection size.

\section{RESULT AND DISCUSSIONS}

Test result showed that among the 14 reflectance targets 13 targets were detected with the automatic target detection method (Figure 7). One reflectance target was not detected in the edge detection step (Figure 8a). To tackle this undetected case, we may adjust threshold for edge detection. However, it could affect false alarms in other cases and was not tried. Figure $8 \mathrm{~b}$ shows reference target candidates extracted by using morphological parameter such as target size and shape. Figure $8 \mathrm{c}$ shows reference target extracted from the candidates in Figure $8 \mathrm{~b}$ using DN variation among bands.

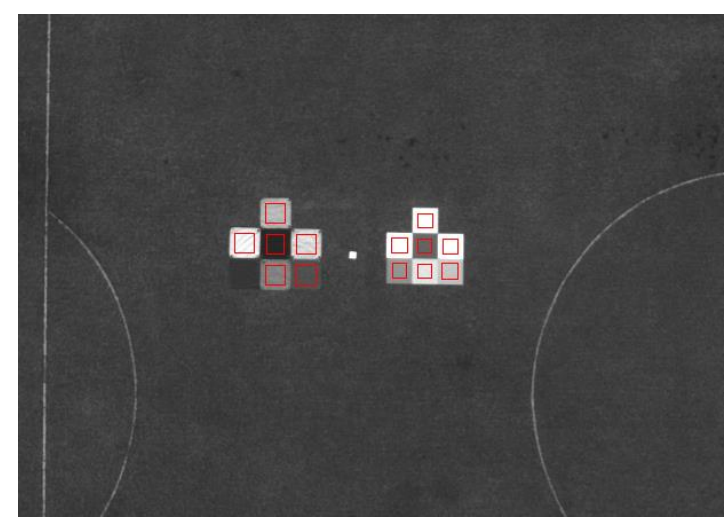

Figure 7. Automatic target detect result.

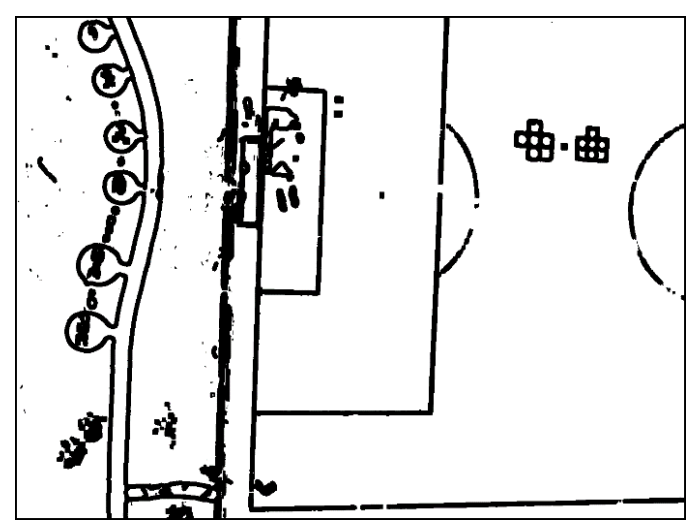

(a)

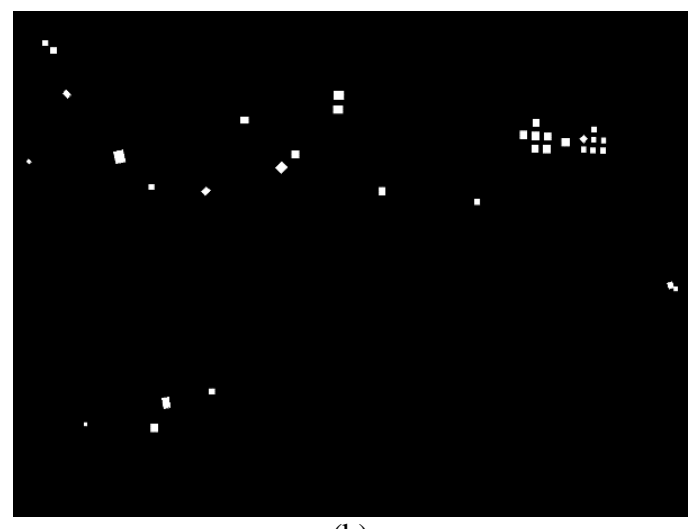

(b) 


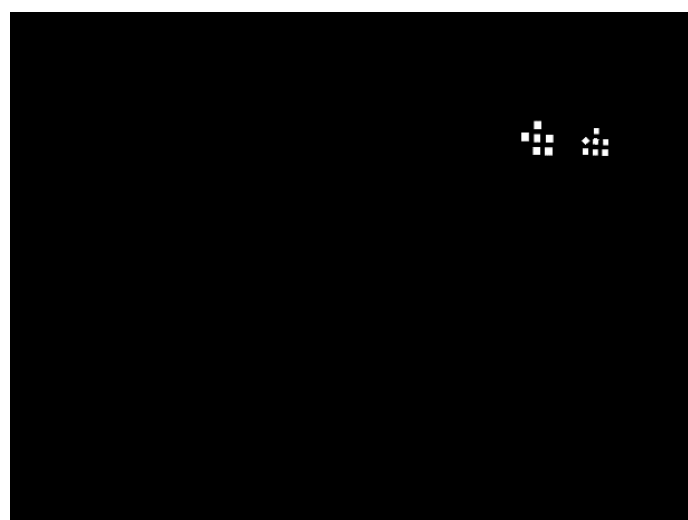

(c)

Figure 8. Result of edge detection.

The undetected target was extracted by semi-automatic target detect method (Figure 9). In addition, test was conducted on the rest target to confirm the semi-automatic target detection method. As a result, other targets were also detected (Figure 10).

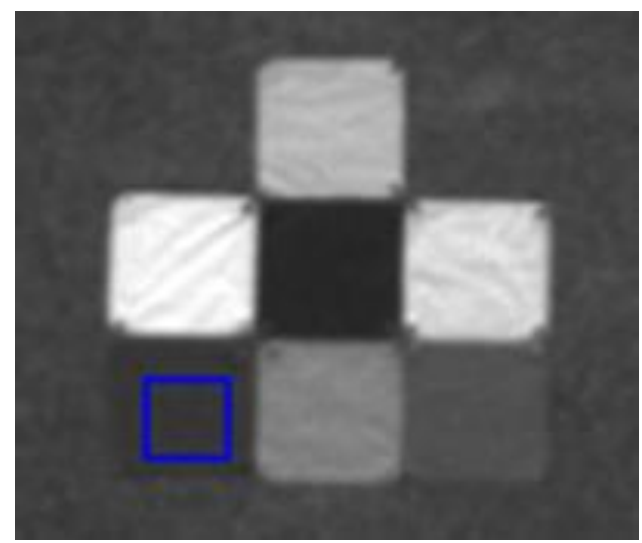

Figure 9. Semi-automatic target detect result.
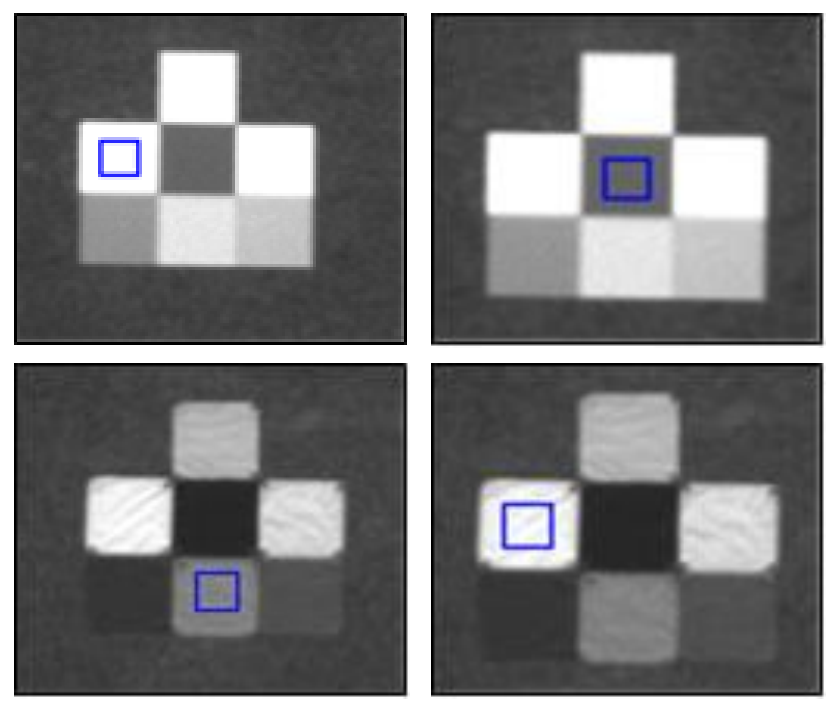

Figure 10. Semi-automatic target detect result.
In other images, false alarms were remained in result of the proposed automatic target detect method. False alarms were used to some artificial targets with high homogeneity. And among them, there are similar in form to reference targets. Therefore, it could not be distinguished by using proposed automatic target detect method (Figure 11). Such false alarms were removed from regression analysis. A user only chose and assigned reflectance values to valid target areas extracted.

\section{CONCLUSIONS}

In this study, we proposed method to automatically detect reflectance targets. To handle undetected targets, we also proposed a semi-automatic target detection. The automatic and semi-automatic target detect methods showed good performance. The target detect method will reduce the time it takes to set target area for vicarious radiometric correction. However, the semi-automatic target detect method only proceeds in four directions, if the targets are not parallel in the image, the detection performance may be degraded. Therefore, further research is needed to handle this issue.

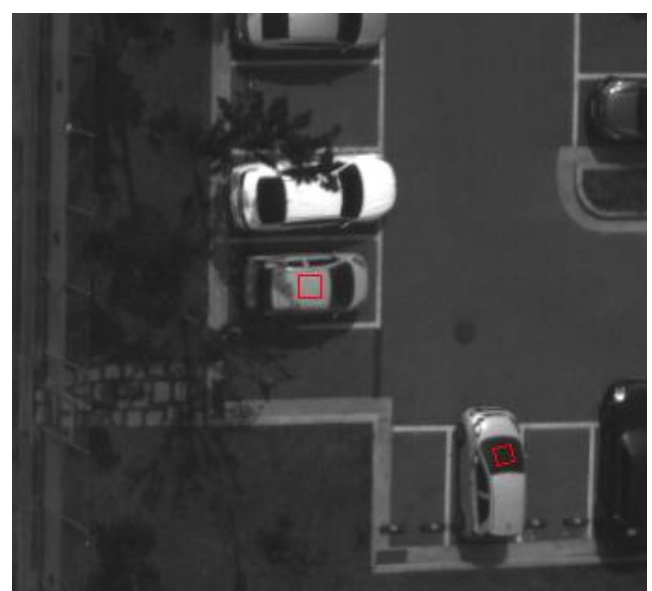

Figure 11. False alarm in the automatic target detect result.

\section{ACKNOWLEDGEMENTS}

This study was carried out with the support of "Cooperative Research Program for Agriculture Science \& Technology Development (PJ01350003)" Rural Development Administration, Republic of Korea and with the support of "National Geographic Information Institute", Republic of Korea.

\section{REFERENCES}

Mathews, A.J., 2015. A Practical UAV Remote Sensing Methodology to Generate Multispectral Orthophotos for Vineyards: Estimation of Spectral Reflectance Using Compact Digital Cameras. International Journal of Applied Geospatial Research, 6 (4), pp. 65-87.

Shin, J.-I.; Cho, Y.-M.; Lee, H.-M.; Lim, P.-C.; Kim, T., 2019. A Ground Reference Target Detection Method For Automatic Vicarious Calibration of UAV Multispectral Image, The $40^{\text {th }}$ Asian Conference on Remote Sensing (ACRS 2019) 
Shin, J.-I.; Cho, Y.-M.; Lim, P.-C.; Lee, H.-M.; Ahn, H.-Y.; Park, C.-W.; Kim, T., 2020. "Relative Radiometric Calibration Using Tie Points and Optimal Path Selection for UAV Images"
Remote
Sensing
12 ,
no.
11:
1726.

https://doi.org/10.3390/rs12111726

Shin, J., Cho, Y., Lee, H., Yoon, S., Ahn, H., Park, C. \& Kim, T. 2020, An Optimal Image Selection Method To Improve Quality Of Relative Radiometric Calibration For UAV Multispectral Images, Copernicus GmbH, Gottingen.

Wang, C. and S.W. Myint, 2015. A Simplified Empirical Line Method of Radiometric Calibration for Small Unmanned Aircraft Systems-Based Remote Sensing, IEEE Journal of Selected Topics in Applied Earth Observations and Remote Sensing, 8 (5), pp. 1876-1885. 